\title{
Domain Formation in DODAB-Cholesterol Mixed Systems Monitored via Nile Red Anisotropy
}

\author{
Graham Hungerford, ${ }^{1}$ Elisabete M. S. Castanheira, ${ }^{1}$ Adelina L. F. Baptista, ${ }^{1}$ Paulo J. G. \\ Coutinho, ${ }^{1}$ and M. Elisabete C. D. Real Oliveira ${ }^{1,2}$
}

Received July 20, 2005; accepted September 19, 2005

Published online: November 24, 2005

\begin{abstract}
The effect of the cholesterol $(\mathrm{Ch})$ on liposomes composed of the cationic lipid dioctadecyldimethylammonium bromide (DODAB) was assessed by studying both the steady-state and time-resolved fluorescence anisotropy of the dye Nile Red. The information obtained combined with analysis of the steady-state emission and fluorescence lifetime of Nile Red (NR) for different cholesterol concentrations (5-50\%) elucidated the presence of "condensed complexes" and cholesterol-rich domains in these mixed systems. The steady-state fluorescence spectra were decomposed into the sum of two lognormal emissions, emanating from two different states, and the effect of temperature on the anisotropy decay of Nile Red for different cholesterol concentrations was observed. At room temperature, the time-resolved anisotropy decays are indicative of NR being relatively immobile (manifest by a high $r_{\infty}$ value). At higher temperature, rotational times ca. 1 ns were obtained throughout and a trend in increasing hindrance was seen with increase of Ch content.
\end{abstract}

KEY WORDS: Cholesterol; DODAB; fluorescence anisotropy; Nile Red.

\section{INTRODUCTION}

An essential feature of the use of vesicles as delivery systems is the conformational instability of their membranes. In cationic vesicles this has been found to be enhanced by inclusion of a "helper" lipid [1-8] although the mechanism responsible for this effect is not fully understood. Zhang and Anchordoquy [9] have studied the relationship between the effect of cholesterol in cationiclipid complexes and the charge density of the lipoplex. There has also been a long-standing interest in the physical chemical properties of mixtures of cholesterol and phospholipids in monolayer and bilayer membranes [1015]. Cholesterol is the major neutral lipid component of biological membranes in most eukaryotic cells and its primary role is that of a modulator of the physical proper-

\footnotetext{
${ }^{1}$ Departamento de Física, Universidade do Minho, Campus de Gualtar, 4710-057 Braga, Portugal.

${ }^{2}$ To whom correspondence should be addressed. E-mail: beta@ fisica.uminho.pt
}

ties of the plasma phospholipid membrane. In one respect cholesterol acts as a "disordering agent," resulting in the broadening of the main phase transition and finally, its disappearance with increasing concentration. On the other hand, it also acts as "ordering agent," causing a reduction in the lipid chain mobility and the average area per lipid molecule $[10,16]$. It is known that cholesterol can eliminate well-defined boundaries that favour bilayer fusion $[17,18]$ and can, at high concentration, form cholesterolrich domains within the lipid bilayer [14] along with condensed complexes or cholesterol crystallites with phospholipids $[13,15,19]$.

In this study we explore the interaction of cholesterol with the long-chain cationic surfactant dioctadecyldimetylammonium bromide (DODAB), which in excess of water, self-assembles above the gel-tocrystalline phase transition temperature $\left(T_{\mathrm{m}}\right)$ into a giant closed bilayer vesicle structure [20-22]. This interaction was monitored using the solvatochromic probe Nile Red. This probe has a hydrophobic nature and has demonstrated its value in the study of biomembrane heterogeneity 
$[23,24]$. Both its steady-state and time-resolved fluorescence emission properties are strongly medium dependent [25-27] and it usually exhibits an increase in fluorescence yield with decreasing solvent polarity, with a corresponding blue shift in the peak emission. By studying the fluorescence anisotropy of Nile Red, we have previously obtained information concerning polarity and hydration level changes in vesicle systems [26].

\section{EXPERIMENTAL}

\section{Materials and methods}

Cholesterol (Ch) was purchased from Sigma. Dioctadecyldimethylammonium bromide (DODAB) was purchased from Tokyo Kasei and the solvatochromic probe Nile Red from Aldrich. All were used as received. Lipid films were prepared from stock solutions of DODAB in chloroform, with $\mathrm{Ch}$ at several molar ratios, by evaporation of solvent under a nitrogen stream. Vesicles were formed by hydration of lipids with an aqueous buffer solution $(20 \mathrm{mM}$ HEPES and $10 \mathrm{mM} \mathrm{NaCl})$, at $60^{\circ} \mathrm{C}$ (above the DODAB transition temperature), followed by sonication in a bath (Heat Systems W-225R), to obtain optically clear solutions. The final DODAB concentration was $1 \mathrm{mM}$. NR $\left(10^{-6} \mathrm{M}\right)$ was introduced to the final vesicle solution by injection of $10 \mu \mathrm{L}$ of a $3 \times 10^{-4} \mathrm{M}$ stock solution. The vesicle solutions were cooled to room temperature and stored for $24 \mathrm{~h}$ prior to measurement.

\section{Fluorescence measurements}

The steady-state fluorescence measurements were performed using Spex Fluorolog 2 spectrofluorimeter. Polarized emission spectra were recorded using GlanThompson polarizers. All spectra were corrected for the instrumental response of the system. The time-resolved fluorescence measurements were performed, both at room temperature $\left(\sim 25^{\circ} \mathrm{C}\right)$ and $55^{\circ} \mathrm{C}$, using a single-photon counting spectrometer equipped with a pulsed nanosecond LED excitation head, with peak emission at $495 \mathrm{~nm}$ (HORIBA, Jobin Yvon, IBH Ltd. Glasgow, Scotland). The detection of the fluorescence, wavelength selected using filters, was made using a Hamamatsu R2949 photomultiplier. The fluorescence lifetime data were measured to 10000 counts in the peak, unless otherwise indicated. The instrumental response function was recorded sequentially using a scattering solution and a time calibration of $23 \mathrm{ps} / \mathrm{channel}$, giving a nominal time resolution of $100 \mathrm{ps}$ after reconvolution. Data were analysed by using a sum of exponentials, employing a nonlinear least squares reconvolution analysis (HORIBA, Jobin Yvon, IBH Ltd. Glasgow, Scotland) of the form,

$$
I(t)=\sum_{i=1}^{n} a_{i} \exp \left(-t / \tau_{i}\right)
$$

The pre-exponential factors $\left(\alpha_{i}\right)$ are shown normalised to 1 and the errors are taken as three standard deviations. The goodness of fit was judged in terms of both a chi-squared $\left(\chi^{2}\right)$ value and weighted residuals. Time-resolved anisotropy decays were analysed

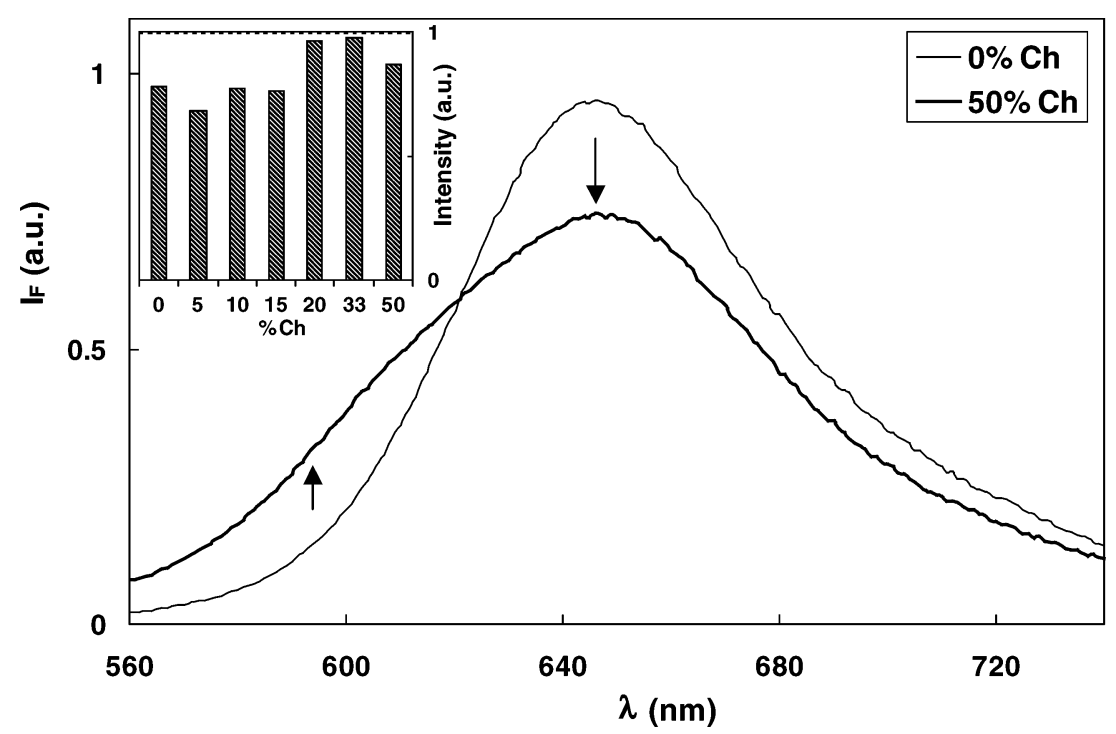

Fig. 1. Emission spectra of Nile Red in DODAB and 1:1 DODAB/Ch vesicles $\left(\lambda_{\text {exc }}=550 \mathrm{~nm}\right)$. Inset: Variation of Nile Red overall emission with increasing cholesterol concentration. 
Table I. Time-Resolved Fluorescence Decay Data for DODAB Vesicles with Added $\mathrm{Ch}$ at Room Temperature $\left(\sim 25^{\circ} \mathrm{C}\right)$

\begin{tabular}{lcccccccc}
\hline$\% \mathrm{Ch}$ & $\tau_{1}$ & $\tau_{2}$ & $\tau_{3}$ & $\alpha_{1}$ & $\alpha_{2}$ & $\alpha_{3}$ & $\tau_{\text {ave }}$ & $\chi^{2}$ \\
\hline 0 & $3.54 \pm 0.08$ & $2.75 \pm 0.45$ & & 0.49 & 0.51 & & 3.1 & 1.11 \\
5 & $6.03 \pm 0.27$ & $2.20 \pm 0.36$ & & 0.40 & 0.60 & & 3.7 & 1.07 \\
33 & $6.33 \pm 0.04$ & & & 1 & & & 6.3 & 1.09 \\
50 & $8.87 \pm 2.70$ & $5.68 \pm 0.96$ & $2.11 \pm 0.81$ & 0.07 & 0.80 & -0.13 & 5.4 & 1.05 \\
\hline
\end{tabular}

Experimental Conditions: (1) Excitation at $490 \mathrm{~nm}$. (2) Emission at $650 \mathrm{~nm}$. (3) Lifetimes measured in nanoseconds.

making use of the impulse response function (IBH DAS6 software).

\section{RESULTS AND DISCUSSION}

In comparison to DODAB alone the emission spectrum of Nile Red in the mixed DODAB-Ch system exhibits a decrease in the peak emission accompanied by a concomitant increase on the blueside of the emission band (manifest as a shoulder), see Fig. 1. This may hint that the NR relocates to a less polar environment. The overall emission intensity, however, remains roughly constant (see inset in Fig. 1) except for a slight increase observed after the addition of $20 \% \mathrm{Ch}$. Related time-resolved fluorescence measurements were performed and the outcome presented in Table I. In most cases the decays are multiexponential and at the highest $\mathrm{Ch}$ concentration a rise time is observed. On increasing $\mathrm{Ch}$ concentration there is a trend for an overall increase in fluorescence lifetime, with a maximum (average lifetime) reached at a concentration of $33 \% \mathrm{Ch}$. These data reflect the changes in the overall emission yield (see inset Fig. 1). The time-resolved values have been linked to the viscosity of the environment and the formation of a solvent relaxed state, with the presence of a biexponential decay and rise times noted [28]. Our data appear consistent with that model, although the need to use the sum of three exponentials indicates that our system is more complex than that previously reported [28]. It should be noted that at room temperature these vesicles are principally in the gel phase and cholesterol apparently distributes evenly between the DODAB lipids, until $\sim 20 \%$, when Ch enrichment leads to complex formation [15]. Overall this would explain the blue shift in the Nile Red emission spectrum as the lipid packing changes and the dye is less accessible to the aqueous region. At the

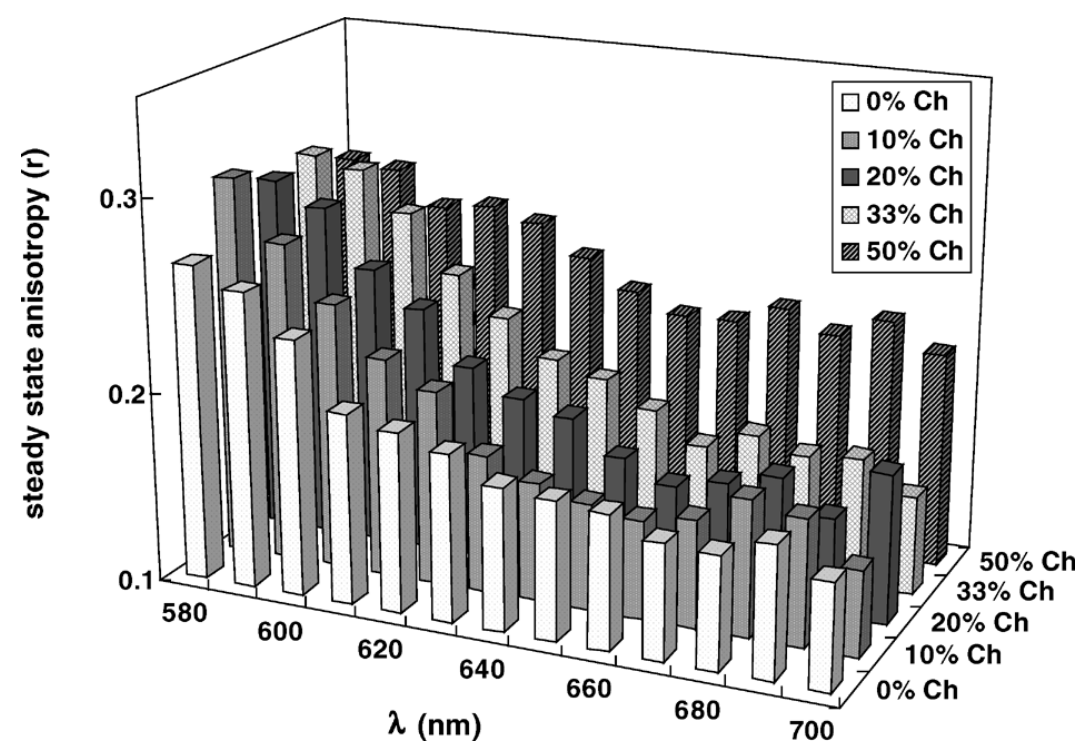

Fig. 2. Variation of Nile Red steady-state anisotropy with emission wavelength for DODAB/cholesterol mixed systems at several cholesterol ratios. 


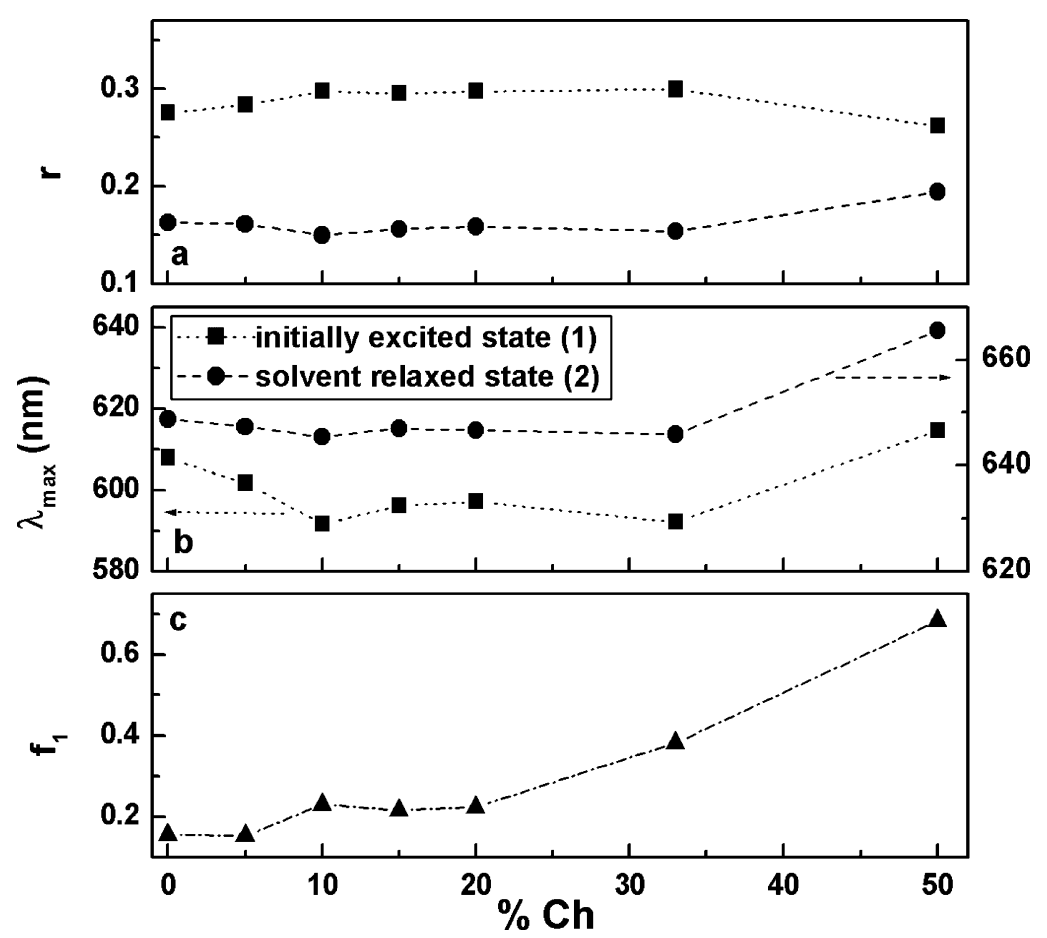

Fig. 3. (a) Calculated steady-state anisotropies for state $1(\boldsymbol{\square})$ and state $2(\boldsymbol{)})$ of Nile Red, as a function of cholesterol ratio. (b) Corresponding emission maxima and (c) fraction of the emission intensity, $f_{1}$, associated with state 1 .

higher Ch concentrations ( $>33 \%$ ) the cholesterol can self associate to form crystalline domains [14], which expel the probe to a more polar environment, hence the overall decrease in lifetime and emission intensity observed at $50 \% \mathrm{Ch}$.

The effect of increasing Ch concentration on the steady-state anisotropy was also noted and the data presented in Fig. 2. This clearly shows trends for increasing anisotropy at lower emission wavelengths and at higher Ch concentrations, consistent with Nile Red packed more tightly in non-polar environments and the addition of $\mathrm{Ch}$ producing a more ordered structure. Further analysis was performed by decomposing the steady-state anisotropy spectra into lognormal components using the method that we have previously described [26]. This ascribes the emission to an initially excited state (state 1) and a solvent relaxed state (state 2) and allows the peak wavelengths, associated anisotropies and the relative fraction of each state to be calculated. These data are presented in Fig. 3. From this figure it is possible to see that the steady-state anisotropies of each state are roughly constant, with a slight increase for state 2 at the highest $\mathrm{Ch}$ concentration. It can also be seen that the major effect is that of the shift in balance of the Nile Red emission from state 2 to state 1 (ca. from 650 to $600 \mathrm{~nm}$ ).

Time-resolved anisotropy measurements were performed to elucidate more information concerning the environment of the Nile Red probe. As well as room

Table II. Time-Resolved Fluorescence Decay Data for DODAB Vesicles with Added Ch at

\begin{tabular}{lcccccccc}
\multicolumn{10}{c}{$55^{\circ} \mathrm{C}$} \\
\hline \%Ch & $\tau_{1}$ & $\tau_{2}$ & $\tau_{3}$ & $\alpha_{1}$ & $\alpha_{2}$ & $\alpha_{3}$ & $\tau_{\text {ave }}$ & $\chi^{2}$ \\
\hline 0 & $3.58 \pm 0.95$ & $2.05 \pm 0.03$ & $0.78 \pm 0.61$ & 0.03 & 0.82 & -0.15 & 1.9 & 1.11 \\
5 & $3.96 \pm 1.50$ & $1.86 \pm 0.03$ & $1.23 \pm 0.66$ & 0.01 & 0.77 & -0.22 & 1.7 & 1.15 \\
33 & $2.94 \pm 0.04$ & $0.85 \pm 0.24$ & & 0.72 & -0.28 & & 2.4 & 1.09 \\
50 & $5.28 \pm 0.09$ & $3.30 \pm 0.12$ & $0.54 \pm 0.39$ & 0.21 & 0.67 & -0.12 & 3.4 & 1.23 \\
\hline
\end{tabular}

Experimental Conditions: (1) Excitation at $490 \mathrm{~nm}$. (2) Emission $>570 \mathrm{~nm}$ except at $33 \% \mathrm{Ch}$ emission at $650 \mathrm{~nm}$. (3) Lifetimes measured in nanoseconds. 

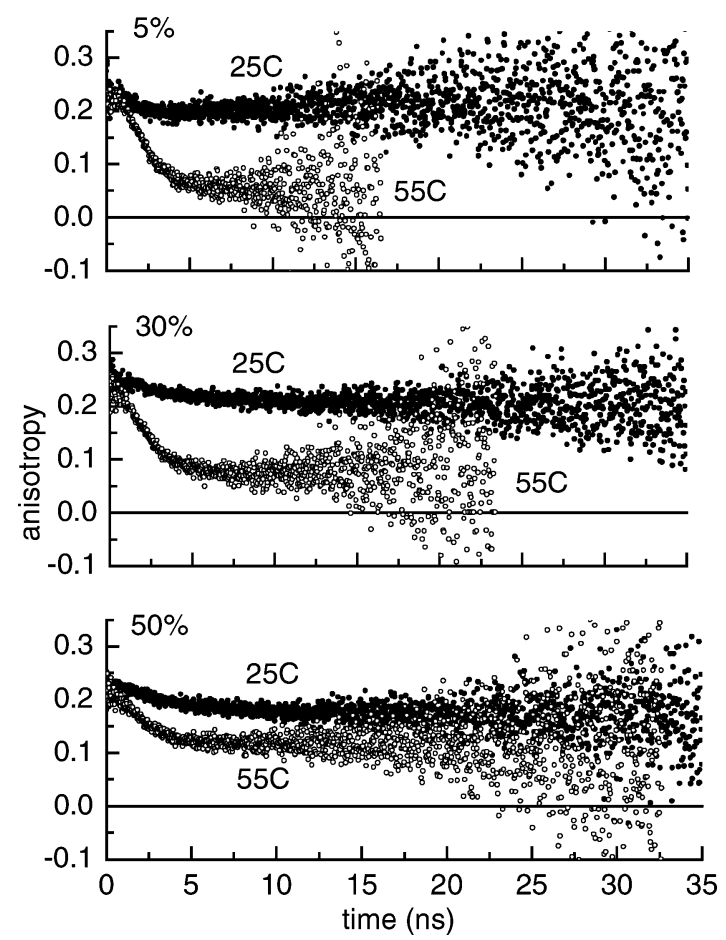

Fig. 4. Time-resolved anisotropy decays for Nile Red in DODAB vesicles $\left(\lambda_{\text {exc }}=500 \mathrm{~nm}\right)$ with different quantities of cholesterol and at two different temperatures, 25 and $55^{\circ} \mathrm{C}$.

temperature measurements (probing the gel phase), measurements were performed at $55^{\circ} \mathrm{C}$ where the mixed systems are in the liquid crystalline phase. Anisotropy decays for Nile Red, excited at $500 \mathrm{~nm}$ with different concentrations of $\mathrm{Ch}$ at room temperature and $55^{\circ} \mathrm{C}$ are given in Fig. 4. Qualitatively it is easy to note (because of the high $r_{\infty}$ value) that the probe rotation is highly hindered at room temperature and that at $55^{\circ} \mathrm{C}$ the effect of Ch enrichment is to increase the hindrance the probe experiences in the liquid crystalline phase. These data are presented in a more quantitative manner in Fig. 5, where the recovered rotational correlation times as well as the semicone angle related to the degree of hindrance is given [29]. At higher temperature, the rotational correlation time is independent of Ch concentration, indicating a uniform viscosity, while that at $25^{\circ} \mathrm{C}$, after an initial decrease, reflects an increase in the viscosity of the probe environment. The rotational freedom of the probe, at both temperatures, is found to decrease with $\mathrm{Ch}$ enrichment and overall the results are consistent with first condensed complex followed by domain formation. Lifetime measurements performed at the higher temperature are presented in Table II. The decays are multiexponential and in general the average lifetimes are $60 \%$ those obtained at room temperature, showing the
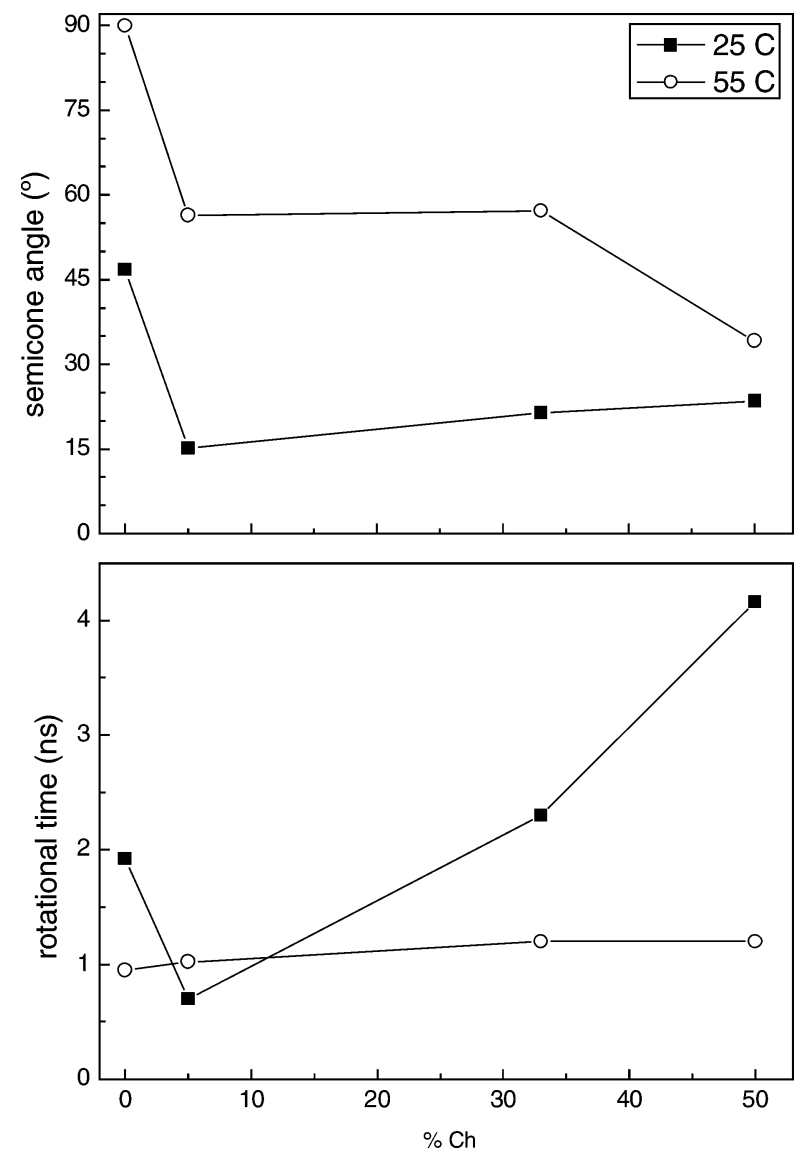

Fig. 5. Time-resolved anisotropy data for DODAB vesicles with increasing concentration of $\mathrm{Ch}$.

increased influence of non-radiative processes. The trend with increasing $\mathrm{Ch}$ concentration is for an increase in decay time after a slight dip on the initial addition of $\mathrm{Ch}$, which reflects the trend in the rotational correlation time and is in agreement with the viscosity dependence of the fluorescence decay time [28].

\section{CONCLUSION}

The effect of the cholesterol distribution, complex and domain formation in mixed DODAB vesicles with cholesterol enrichment can be ascertained via the use of both steady-state and time-resolved anisotropy. The Nile Red fluorescence was able to provide information concerning both the polarity and viscosity of these mixed vesicles, which is important in understanding their use as delivery systems. 


\section{REFERENCES}

1. J. H. Felgner, R. Kumar, C. N. Sridhar, C. J. Wheeler, Y. J. Tsai, R. Border, P. Ramsey, M. Martim, and P. L. Felgner (1994). Enhanced gene delivery and mechanism studies with a novel series of cationic lipid formulations. J. Biol. Chem. 269, 2550-2561.

2. D. D. Lasic (1995). In D. D. Lasic and Yechezkel Barenholz (Eds.), Handbook of Nonmedical Applications of Liposomes: From Gene Delivery and Diagnostic to Ecology, CRC Press, New York, Vol. IV, pp. 1-32.

3. D. D. Lasic and D. Ruff (1998). In D. D. Lasic and D. Papahadjoupoulos (Eds.), Medical Applications of Liposomes, Elsevier, Netherlands, pp. 353-394.

4. R. B. Campbell, V. Sathyamangalam, Balasubramanian, and R. M. Straubinger, (2001). Phospholipid-cationic lipid interactions: Influences on membrane and vesicle properties. Biochim. Biophys. Acta 1512, 27-39.

5. D. Hirsch-Lerner and Y. Barenholz (1999). Hydration of lipoplexes commonly used in gene delivery: Follow-up by Laurdan fluorescence changes and quantification by differential scanning calorimetry. Biochim. Biophys. Acta 1461, 47-57.

6. S. J. Eastman, C. Siegel, J. Tousignant, A. E. Smoth, S. H. Cheng, and R. K. Scheule (1997). Biophysical characterization of cationic lipid:DNA complexes. Biochim. Biophys. Acta 1325, 41-62.

7. L. Ciani, S. Ristori, A. Salvati, L. Calamai, and G. Martini (2004). DOTAP/DOPE and DC-chol/DOPE lipoplexes for gene delivery: Zeta potential measurements and electron spin resonance spectra. Biochim. Biophys. Acta 1664, 70-79.

8. Y. S. Mel'nikova, S. M. Mel'nikov, and J.-E. Löfroth (1999). Physico-chemical aspects of the interaction between DNA and oppositely charged mixed liposomes. Biophys. Chem. 81, 125-141.

9. Y. Zhang and T. J. Anchordoquy (2004). The role of lipid charge density in the serum stability of cationic lipid/DNA complexes. Biochim. Biophys. Acta 1663, 143-157.

10. P. L. Yeagle (1988) The Biology of Cholesterol, CPC Press, Boca Raton, FL, pp. 242.

11. A. M. Samuni, A. Lipman, and Y. Barenholz (2000). Damage to liposomal lipids: Protection by antioxidants and Cholestrol-mediated dehydration. Chem. Phys. Lipids 105, 121-134.

12. S. L. Veatch and S. L. Keller (2002). Organization in lipid membranes containing cholesterol. Phys. Rev. Lett. 26, 268101-1268101-4.

13. D. Bach and E. Wachtel (2003). Phospholipid/cholesterol model membranes: formation of cholesterol crystallites. Biochim. Biophys. Acta 1610, 187-197.

14. R. Preston Mason, T. N. Tulenko, and R. F. Jacob (2003). Direct evidence for cholesterol crystalline domains in biological mem- branes: role in human pathobiology. Biochim. Biophys. Acta 1610, 198-207.

15. H. M. McConnell and A. Radhakrishnan (2003). Condensed complexes of cholesterol and phospholipids. Biochim. Biophys. Acta 1610, 159-173.

16. A. Genz, F. Holzwarth, and T. Tsong (1986). The influence of cholesterol on the main phase transition of unilamellar dipalmitoylphosphatidylcholine vesicles. A differential scanning calorimetry and iodine laser T-jump study. Biophys. J. 50, 1043-1051.

17. G. Cevc, and H. Richardsen (1999). Lipid vesicles and membrane fusion. Adv. Drug Delivery Rev. 38, 207-232.

18. A. L. Baley and P. R. Cullis (1997). Membrane fusion with cationic liposomes: Effects of target membrane lipid composition. Biochemistry 36, 1628-1634.

19. S. Koronkiewicz and S. Kalinowski (2004). Influence of cholesterol on electroporation of bilayer lipid membranes: Chronopotentiometric studies. Biochim. Biophys. Acta 1661, 196-203.

20. A. M. Carmona-Ribeiro (1992). Synthetic amphiphile vesicles Chem. Soc. Rev. 21, 209-214.

21. E. Feitosa, P. C. A. Barreleiro, and G. Olofsson (2000). Phase transition in dioctadecyldimethylammonium bromide and chloride vesicles prepared by different methods. Chem. Phys. Lipids 105, 201213.

22. P. C. A. Barreleiro (2001). Equilibrium and Kinetic Studies of the Binding of DNA to Cationic Lipids, $\mathrm{PhD}$ Thesis, University of Lund, Lund, Sweden.

23. O. G. Mouritsen and K. Jorgensen (1997). Small scale lipid membrane structure. Current Opin. Struct. Biol. 7, 518-527.

24. Ira and G. Krishnamoorthy (2001). Probing the link between proton transport and water content in lipid membranes. J. Phys. Chem. B 105, 1484-1488.

25. G. Hungerford, E. M. S. Castanheira, M. E. C. D. Real Oliveira, M. G. Miguel, and H. D. Burrows (2002). Monitoring ternary systems of $\mathrm{C}_{12} \mathrm{E}_{5} /$ water/tetradecane via fluorescence of solvatochromic probes. J. Phys. Chem. B 106, 4061-4069.

26. P. J. G. Coutinho, E. M. S. Castanheira, M. C. Rei, and M. E. C. D. Real Oliveira, (2002). Nile Red and DCM fluorescence anisotropy studies in $\mathrm{C}_{12} \mathrm{E}_{7}-\mathrm{DPPC}$ mixed systems. J. Phys. Chem. B 106, 12841-12846.

27. Ira and G. Krishnamoorthy (2001). Fluorescence lifetime distribution in characterizing membrane heterogeneity. J. Fluoresc. 11 247-253.

28. M. M. G. Krishna (1999). Excited-state kinetics of the hydrophobic probe Nile Red in membranes and micelles. J. Phys. Chem. A 103, 3589-3595.

29. R. Steiner (1999). In J. R. Lakowicz (Ed.), Topics in Fluorescence Spectroscopy, Vol. 2, Plenum Press, New York, pp. 1-52. 